\title{
What caused the rapid recovery of the Carrington storm?
}

Kunihiro Keika ${ }^{1 *}$, Yusuke Ebihara ${ }^{2}$ and Ryuho Kataoka ${ }^{3}$

\begin{abstract}
The geomagnetic storm during the Carrington event, which occurred on 2 September 1859, displayed extremely rapid recovery. The geomagnetic field increased by approximately $650 \mathrm{nT} / \mathrm{h}$ at Bombay, India, and by $>300 \mathrm{nT} / \mathrm{h}$ in 1 -h averaged data. Although the rapid recovery is considered due to a sudden increase in the magnetopause current, a sudden decrease of the ring current, or/and a sudden enhancement of the ionospheric currents, this study focuses on the ring current decay. The Carrington rapid recovery had a time constant (approximately $1 \mathrm{~h}$ ) comparable to the storm development (i.e., decrease in the geomagnetic field), indicating that energy loss from the ring current region is predominantly controlled by $E \times B$ convection transport which is responsible for energy input during the storm main phase. This feature has led us to a hypothesis that the flow-out of dense ring current ions and injections of tenuous plasma sheet ions caused the rapid decay of the ring current and in turn the storm rapid recovery. This study examines whether the Carrington rapid recovery can be explained by the flow-out effect. We extend the empirical Burton's model to a model that takes into consideration a sudden change in solar wind density which is correlated with plasma sheet density. We first apply the extended Burton's model to previously observed four intense magnetic storms (Dst minimum <-200 nT) for which solar wind data are available. Using the best fit parameters found by forward modeling, the extended model estimates the recovery of the Carrington storm. The estimate indicates that a solar wind structure with a density bump by approximately $100 \mathrm{~cm}^{-3}$ (and southward interplanetary magnetic field (IMF) of $65 \mathrm{nT}$ and solar wind speed of 1,500 km/s) can cause the rapid recovery under a continuous southward IMF condition. We conclude that the flow-out effect plays a significant role in producing the rapid recovery of the Carrington storm.
\end{abstract}

Keywords: Intense magnetic storms; Ring current; Storm rapid recovery; Flow-out loss; Carrington event; Plasma sheet density change

\section{Background}

A magnetic storm is characterized by a negative excursion of the geomagnetic field at low latitudes (e.g., Gonzalez et al. 1994). As a geomagnetic storm is a global phenomenon, its development and recovery are well represented by a decrease of the disturbance field (Dst) index, which is derived by averaging the geomagnetic field observed by four stations located at low latitudes (http://wdc.kugi.kyoto-u.ac.jp/dstdir/index.html). Dst decreases with increasing geomagnetic activity; it reaches -30 to $-100 \mathrm{nT}$ at minimum for small and moderate storms. In response to a powerful/large coronal mass ejection (CME)

\footnotetext{
*Correspondence: kkeika@stelab.nagoya-u.ac.jp

'Solar-Terrestrial Environment Laboratory, Nagoya University, Nagoya 464-8601, Japan

Full list of author information is available at the end of the article
}

or high-speed solar wind stream, an intense storm with Dst decreasing down to $<-100 \mathrm{nT}$ can occur.

A magnetic storm recovers to the pre-storm level within several to 10 days. As far as the geomagnetic storms that have ever been observed, the recovery is more rapid for an intense storm than for a small/moderate storm (e.g., Yokoyama and Kamide 1997). Most of intense storms and some of moderate storms show a two-step recovery with a quick Dst increase followed by a slow increase to the pre-storm level. These characteristics indicate that the storm recovery is controlled by at least two or several physical processes that occur on different time scales.

On 1 September 1859, a huge solar flare was observed by Carrington (1859) and Hodgson (1859). About $17 \mathrm{~h}$ and 40 min later, a magnetogram installed at Bombay, India,

\section{空}


exhibited an extremely large depression (approximately 1,600 nT in 2 h) (e.g., Tsurutani 2003; Siscoe et al. 2006). The extremely intense geomagnetic storm, known as the most intense storm ever recorded, called the Carrington storm, recovered by $>1,300 \mathrm{nT}$ in $2 \mathrm{~h}$. The top panel of Figure 1 shows the time profile of the geomagnetic field at Bombay, India (after Siscoe et al. (2006), modified from Tsurutani et al. (2003)). Even the time profile of the 1-h averaged data shows that an approximately 850-nT decrease followed by an approximately 600-nT increase occurred on similar time scales. The rapid recovery preceded a several-hour-long slow recovery, and after a relatively small enhancement of another storm activity, a day-long slow recovery back to the pre-storm level.

It is well accepted that the global geomagnetic field disturbance predominantly results from changes in intensity of the current flowing in the Earth's inner magnetosphere, called the ring current, which is mainly controlled by the ion pressure and its spatial gradient (e.g., Ebihara and Ejiri 2003 and references therein). The ion pressure in the core ring current region (i.e., $3<r<8 \mathrm{R}_{\mathrm{E}}$, where $r$ is the geocentric distance) is dominated by ions with energies of 1 to a few $100 \mathrm{~s} \mathrm{keV} \mathrm{(e.g.,} \mathrm{Williams} \mathrm{1981).} \mathrm{Loss} \mathrm{of}$ those energetic ions results in pressure decrease and in
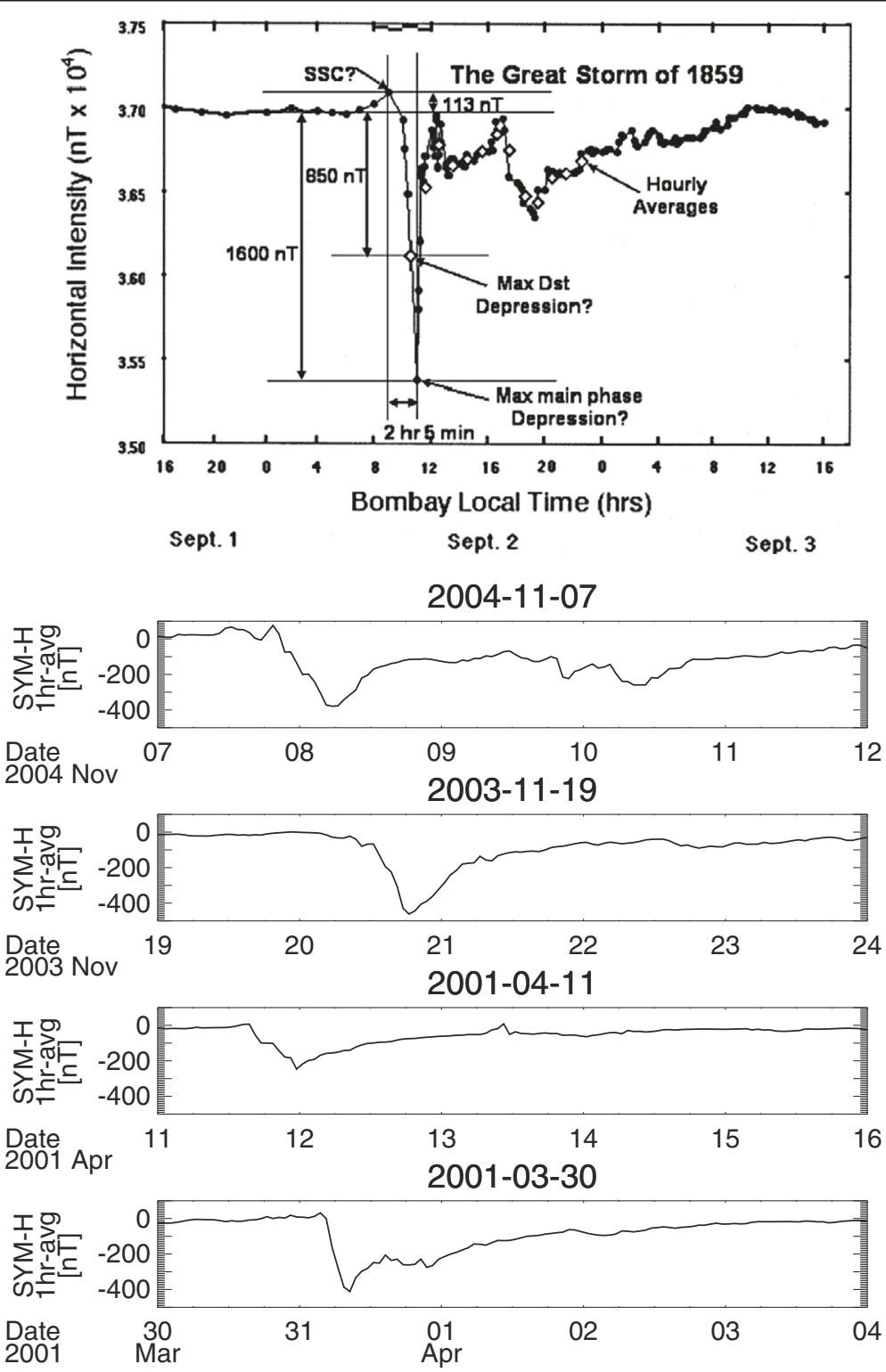

Figure 1 Geomagnetic field variations for the Carrington event and the intense storms examined. The top panel shows geomagnetic field variations at Bombay, India, during the Carrington event (after Siscoe et al. (2006), modified from Tsurutani et al. (2003)). The bottom four panels present 1-h averaged SYM-H index for the intense storms examined in this study. SYM-H, symmetric disturbance field in the horizontal direction. 
turn ring current decay and consequently causes recovery from the depression of the geomagnetic field.

A large number of observational and theoretical studies have investigated physical processes that can explain the storm rapid recovery. It remains unresolved what process dominates over the others, although the slow recovery is well recognized as a result of charge exchange collisions between ring current protons and low-energy neutrals called the geocorona. The major processes that could contribute most to the rapid recovery are (1) charge exchange loss of singly charged oxygen ions $\left(\mathrm{O}^{+}\right)$ (e.g., Hamilton et al. 1988; Daglis et al. 1999); (2) flow-out of ring current ions through the dayside magnetopause, under the conditions of continued/weakened convection where newly injected ions from the plasma sheet are more tenuous than those leaking out into the magnetosheath (e.g., Liemohn 1999, 2001; O'Brien et al. 2002; Keika et al. 2005); (3) pitch angle scattering of ring current ions in the stretched near-Earth magnetotail with small curvature and consequent precipitation into the atmosphere (e.g., Ebihara et al. 2011); and (4) pitch angle scattering of ring current ions through resonant interactions with electromagnetic ion cyclotron (EMIC) waves and consequent precipitation into the atmosphere (e.g., Cornwall et al. 1970; Kozyra et al. 1998a; Jordanova et al. 2001). The details of the processes and related previous studies are summarized in a review paper by Ebihara and Ejiri (2003).

The rapid recovery of the Carrington event (see Figure 1) remains an open question. Siscoe et al. (2006) and Li et al. (2006) calculated Carrington's recovery from the results of their simulation using a solar wind structure that could have caused the Carrington storm development; they made use of an empirical formula, Burton's model (Burton et al. 1975). The estimated recovery was much slower than the observed one. Aguado et al. (2010) proposed a hyperbolic model of storm recovery, and Cid et al. (2013) applied the model to intense storms including the Carrington storm. They showed high accuracy of the hyperbolic function to reproduce the rapid recovery. Aguado et al. (2010) and Cid et al. (2013) commented on the existence of diverse processes responsible for the two-step recovery. However, they did not mention about the relative significance of ion loss processes that could occur in the inner magnetosphere. $\mathrm{Li}$ et al. (2006) introduced a sudden enhancement of the solar wind density that starts at the time of the storm maximum. The density enhancement, which enhances the magnetopause current, produces recovery from the storm development. They suggested that a density sudden increase up to $1,800 \mathrm{~cm}^{-3}$ can reproduce the observed rapid recovery. Such an extreme solar wind density enhancement is, however, unrealistic because it is 1 order magnitude higher than the observed limit at $1 \mathrm{AU}$ distance.
In this paper, we examine whether the Carrington rapid recovery can be explained by the flow-out effect associated with changes in the plasma sheet density (i.e., leakage of denser ions and injections of more tenuous ions), by extending Burton's model and applying it to previously observed intense storms. There have been a large number of modeling studies that demonstrated a significant role of plasma sheet density in variations of the ring current intensity and the storm magnitude. A dense plasma sheet during a period of enhanced convection results in higher ion pressure in the inner magnetosphere and in turn stronger ring current (e.g., Chen et al. 1994; Kozyra et al. 1998b; Ebihara and Ejiri 2000; Ebihara et al. 2005).

A storm recovery due to the flow-out effect can be explained mostly by the rate of plasma sheet density change and the drift speed of energetic ions. A density change determines how much the magnetic field magnitude changes, and the ion drift speed determines the time scale of the magnetic field change. The ion drift is mostly controlled by $\mathbf{E} \times \mathbf{B}$ drift for both ion injections and flow-out. The Carrington event consists of the rapid main phase and rapid recovery phase, both of which have comparable time constants (approximately $1 \mathrm{~h}$ ). This feature indicates that ion transport during the rapid recovery is controlled by a process that dominates ion transport during the main phase, namely, the $\mathbf{E} \times \mathbf{B}$ drift. We thus hypothesize that the ion flow-out effect is a plausible process that causes the rapid recovery.

The fact that the Carrington event recovered on a similar time scale to its development concerns us about possible enhancements of strong ground-induced currents (GIC) (e.g., Pulkkinen et al. 2012) even for the recovery phase. GIC could cause severe impact on our modern technological infrastructure such as pipelines and power grids as occurred during the 13 March 1989 storm (Bolduc 2002). Rapid recovery is thus a space weather issue that needs to be resolved like large geomagnetic sudden commencements (Araki 2014).

In the 'Analysis and results' section, we extend Burton's model to take into consideration the effects of solar wind density changes on storm development and decay. We then apply the extended model to four intense magnetic storms that occurred in years 2001, 2003, and 2004. The 1-h averaged symmetric disturbance field in the horizontal direction (SYM-H) during the storms is presented in Figure 1. For each event, by forward modeling, we find a pair of the model parameters that best fits the observed SYM-H temporal profile. We then apply the model to the Carrington event, using solar wind drivers estimated/used in several previous papers and the best fit parameters derived for the four intense storms. We present the results and discuss about preferred conditions for the flow-out process and other 
significant processes in the 'Results and discussion' section. We summarize our conclusions in the 'Conclusions' section.

\section{Methods}

\section{Extended Burton's model}

In order to examine the flow-out effects on the storm rapid recovery, we extend Burton's empirical model by adding a new term that represents a change in the solar wind density. Temporal variations of the Dst index based on the original Burton's equation (Burton et al. 1975) are expressed as:

$$
\frac{d \text { Dst }_{0}}{d t}=Q-\frac{1}{\tau} \text { Dst }_{0},
$$

where Dst $t_{0}$ is the corrected Dst for which the contributions from the current other than the ring current are subtracted. In this study, we subtracted three types of currents: the magnetopause current, the tail current, and the inductive current on/under the ground. It is well accepted that the contribution from the magnetopause current is proportional to the square root of the solar wind dynamic pressure (e.g., Siscoe et al. 1968). The relationship is expressed as:

$$
\text { Dst }_{0}=\text { Dst }-b \cdot \sqrt{P_{d}}+c,
$$

where $b$ and $c$ are constants. In this study, we use $b=7.26$ $\mathrm{nT} / \mathrm{nPa}^{1 / 2}$ and $c=11.0 \mathrm{nT}$, which were derived by O'Brien and McPherron (2000). The contributions from the ground-induced current and the tail current have been controversial issues (e.g., Langel and Estes 1985; Alexeev et al. 1996; Turner et al. 2000). In this study, we assume $25 \%$ contributions from both currents, taking numbers close to the estimates by Langel and Estes (1985) and Turner et al. (2000).

The energy input term, $Q$, of Equation 1 is defined as:

$$
Q[\mathrm{nT} / \mathrm{h}]=\left\{\begin{array}{cl}
a\left(V B_{\mathrm{S}}-E_{\mathrm{c}}\right) & V B_{\mathrm{S}} \geq E_{\mathrm{c}} \\
0 & V B_{\mathrm{S}}<E_{\mathrm{c}}
\end{array},\right.
$$

where $a$ and $E_{\mathrm{c}}$ are constants, and $V$ and $B_{\mathrm{s}}$ are the solar wind speed and southward component of the interplanetary magnetic field (IMF), respectively. $E_{\mathrm{c}}$ was estimated as $0.49 \mathrm{mV} / \mathrm{m}$ by O'Brien and McPherron (2000).

$\tau$ is the recovery time constant. The Dst recovery rate represented by the loss term increases with increasing Dst, indicating that the loss process(es) considered here is(are) more effective as a storm develops and in turn the plasma pressure in the inner magnetosphere increases. Charge exchange loss can be categorized in this group. Previous studies showed that a constant value of $\tau$ cannot reproduce the rapid recovery and two-step recovery of intense storms (e.g., Hamilton et al. 1988; Gonzalez et al. 1989; Liemohn et al. 1999; Monreal
MacMahon and Llop-Romero 2008). In this study, we choose $\tau$ of 1 day to represent charge exchange collisions of $<50 \mathrm{keV}$ protons or approximately $100 \mathrm{keV} \mathrm{O+}$ ions with the geocorona at $L=3$ to 4 (Ebihara and Ejiri 2003). This study focuses most on evaluating how quickly the flow-out effect can recover the Carrington storm. For simplicity, we take into consideration the most general, best accepted loss process, that is, charge exchange of energetic ( 1 to a few $100 \mathrm{~s} \mathrm{keV}$ ) ions.

The loss term of the original Burton's model assumes the rate of Dst recovery proportional to the plasma pressure in the inner magnetosphere. However, a change in the plasma sheet density, which is the key phenomenon that makes the flow-out process effective, is independent of plasma pressure already stored in the inner magnetosphere. The rate of plasma sheet density variations and the drift speed of energetic ions determine the rate of storm recovery. Thus, in order to describe the flow-out effect, we need to add a new term that changes as a function of plasma sheet density variations and the global convection electric field. Since the plasma sheet density and the convection electric field are well correlated with the solar wind density and electric field, respectively (Terasawa et al. 1997; Borovsky et al. 1998; Ebihara and Ejiri 2000), we propose an extended Burton's model as:

$$
\frac{d \mathrm{Dst}_{0}}{d t}=Q-\frac{1}{\tau} \mathrm{Dst}_{0}+\Psi
$$

where $\tau$ is the time constant; the input term, $Q$, is the same as the original Burton's model:

$$
Q=\left\{\begin{array}{cc}
\alpha\left(V B_{\mathrm{S}}-E_{\mathrm{c}}\right) & V B_{\mathrm{S}} \geq E_{\mathrm{c}} \\
0 & V B_{\mathrm{S}}<E_{\mathrm{c}}
\end{array}\right.
$$

and the new term, $\Psi$, is expressed as:

$$
\Psi=\left\{\begin{array}{rl}
\beta \cdot\left(V B_{\mathrm{S}}-E_{\mathrm{c}}\right) \cdot \frac{d N_{\mathrm{SW}}}{d t} & V B_{\mathrm{S}} \geq E_{\mathrm{c}} \\
0 & V B_{\mathrm{S}}<E_{\mathrm{c}}
\end{array}\right.
$$

where $N_{\mathrm{SW}}$ is the solar wind density, $B_{\mathrm{s}}$ is the IMF southward component, and $E_{\mathrm{c}}$ is a constant. The time variation of $N_{\mathrm{SW}}$ is defined as:

$$
\frac{d N_{\mathrm{SW}}(t)}{d t}=\frac{d\left\{N_{\mathrm{SW}}\left(t-\Delta t_{\mathrm{r}}\right)-N_{\mathrm{SW}}\left(t-\Delta t_{\mathrm{r}}-\Delta t_{\mathrm{d}}\right)\right\}}{d t},
$$

where $\Delta t_{\mathrm{r}}$ is the time required for the plasma sheet density to respond to changes in the solar wind density.

$\Delta t_{\mathrm{d}}$ is chosen as the typical time required for ring current ions to drift from the near-Earth magnetotail to the dayside magnetopause. When energetic ions are injected into the inner magnetosphere (i.e., crossed the outer edge of the inner magnetosphere on the night side) at $t=t_{0}$, the injected ions drift to the dayside 
magnetopause for a time period of $\Delta t$ and eventually flow out through the dayside magnetopause at $t=t_{0}+$ $\Delta t_{\mathrm{d}}$. What affects the ring current intensity is the difference in density between the outflowing ring current ions and ions newly injected from the plasma sheet at $t=t_{0}+$ $\Delta t_{\mathrm{d}}$. Thus, for a Dst change at time $t$, the density of the outflowing ring current ions and the injected ions are correlated with the solar wind density at $t=t-\Delta t_{\mathrm{r}}-\Delta t_{\mathrm{d}}$ and $t=t-\Delta t_{\mathrm{r}}$. In this study, we chose $\Delta t_{\mathrm{d}}=4 \mathrm{~h}$.

\section{Application to observed intense storms}

We surveyed intense storm events during which the solar wind and IMF were monitored. We found four events, the first three of which are listed in the top tenth intense storms recorded since year 1957. The bottom four panels of Figure 1 present the time profile of the 1-h averaged SYM-H index for the four storms. We used the SYM-H index instead of Dst index because it has the same time resolution as the OMNI data. The minimum of 1-h averaged SYM-H is $-379.3 \mathrm{nT}$ for the November 2004 storm, $-461.3 \mathrm{nT}$ for the November 2003 storm, -246.3
nT for the April 2001 storm, and $-412.0 \mathrm{nT}$ for the March 2001 storm. We used 1-h averaged OMNI solar wind data for the parameters required for the extended Burton's model, specifically solar wind density, speed, and IMF north-south component.

\section{Search of the best fit parameters}

We performed forward modeling to find a set of the parameters $(\alpha$ and $\beta$ ) of Equations 5 and 6 that best matches our extended model to the 1-h averaged SYM$\mathrm{H}$ profile of each storm. Our forward modeling takes the following steps.

A. The SYM-H time profile based on our extended model (Equation 4) is calculated for different parameter pairs $\left[\alpha_{\mathrm{i}}, \beta_{\mathrm{i}}\right] . \alpha_{\mathrm{i}}$ is chosen from -5.0 to -0.1 with 0.1 increment; $\beta_{\mathrm{i}}$ is chosen from -0.1 to 0.0 with 0.01 increment.

B. $\left[\alpha_{\mathrm{j}}, \beta_{\mathrm{j}}\right]$ pairs are found that provide the SYM-H minimum within $5 \mathrm{nT}$ of the observed SYM-H minimum.

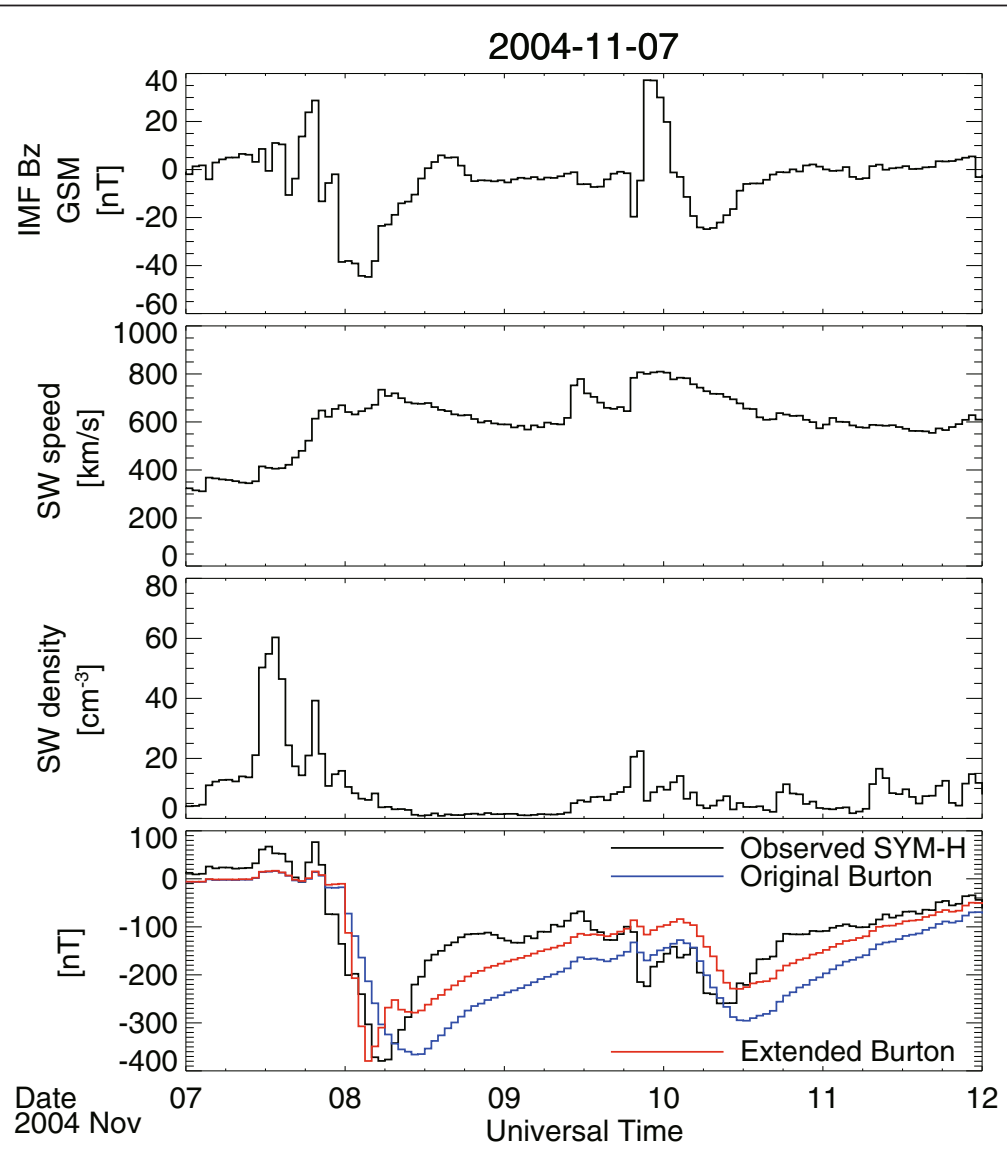

Figure 2 Solar wind speed, solar wind density, IMF Bz, and 1-h averaged SYM-H for the November 2004 storm. The bottom panel also includes 1-h SYM-H derived from the original Burton's model (blue line) and 1-h SYM-H derived from our extended Burton's model (red line). IMF, interplanetary magnetic field; Bz, the Z component of the magnetic field; GSM, geocentric solar magnetospheric; SYM-H, symmetric disturbance field in the horizontal direction. 
C. The pair $\left[\alpha_{0}, \beta_{0}\right]$ that minimizes the mean square $\left(\sigma^{2}\right)$ of the differences between the modeled SYM-H and the observed SYM-H is selected from the $\left[\alpha_{\mathrm{j}}, \beta_{\mathrm{j}}\right]$ pairs. $\sigma^{2}$ is calculated as:

$$
\sigma^{2}=\sum_{t=t_{\text {sart }}}^{t_{\text {end }}}\left\{\mathrm{SYM}-\mathrm{H}(t)_{\text {modeled }}-\mathrm{SYM}-\mathrm{H}(t)_{\text {observed }}\right\}^{2}
$$

where $t_{\text {start }}$ and $t_{\text {end }}$ are the start and end times of a storm interval of interest, respectively. In this study, we defined $t_{\text {start }}$ as $00 \mathrm{UT}$ of the storm start date and $t_{\text {end }}$ as 24 UT of the storm end date which we chose as 5 days after the start date.

We conducted the same analysis for different values of $\Delta t_{\mathrm{r}}$. We chose $\Delta t_{\mathrm{r}}$ from 0 to $5 \mathrm{~h}$ with 1-h increment for the November 2003, April 2001, and April 2003 events. We chose $\Delta t_{\mathrm{r}}$ from 10 to $20 \mathrm{~h}$ with 1-h increment for the November 2004 event because it is apparent that a $>10$-h time lag is needed for the solar wind density decrease to account for the storm rapid recovery. The parameter pair $\left[\alpha_{0}, \beta_{0}\right]$ and $\Delta t_{\mathrm{r}}$ that returns the smallest $\sigma^{2}$ are regarded as the best fit.

\section{Results and discussion}

Figure 2 presents IMF $\mathrm{Z}$ component of the magnetic field $(\mathrm{Bz})$ in GSM coordinates, solar wind speed, solar wind density, and observed SYM-H, all of which are averaged over $1 \mathrm{~h}$, for the November 2004 storm. The bottom panel also shows SYM-H derived from the original Burton's model (blue line) and SYM-H derived from our extended model (red line) with the best fit parameters. We chose the constant $a$ of Equation 3 so that the original Burton's model is consistent with the observed SYM-H at its minimum. Data and model results for the November 2003 storm, the April 2001 storm, and the March 2001 storm are shown in Figures 3, 4, and 5, respectively, in the same format as Figure 2. All the storms were caused mostly by an increase of solar wind speed and a southward excursion of IMF (and in turn an increase of the dawn-dusk electric field). The pair of the best fit parameters $\alpha_{0}, \beta_{0}, \Delta t_{\mathrm{r}}$ is listed in Table 1, along with the SYM-H minimum, the IMF Bz minimum, and the solar wind density maximum.

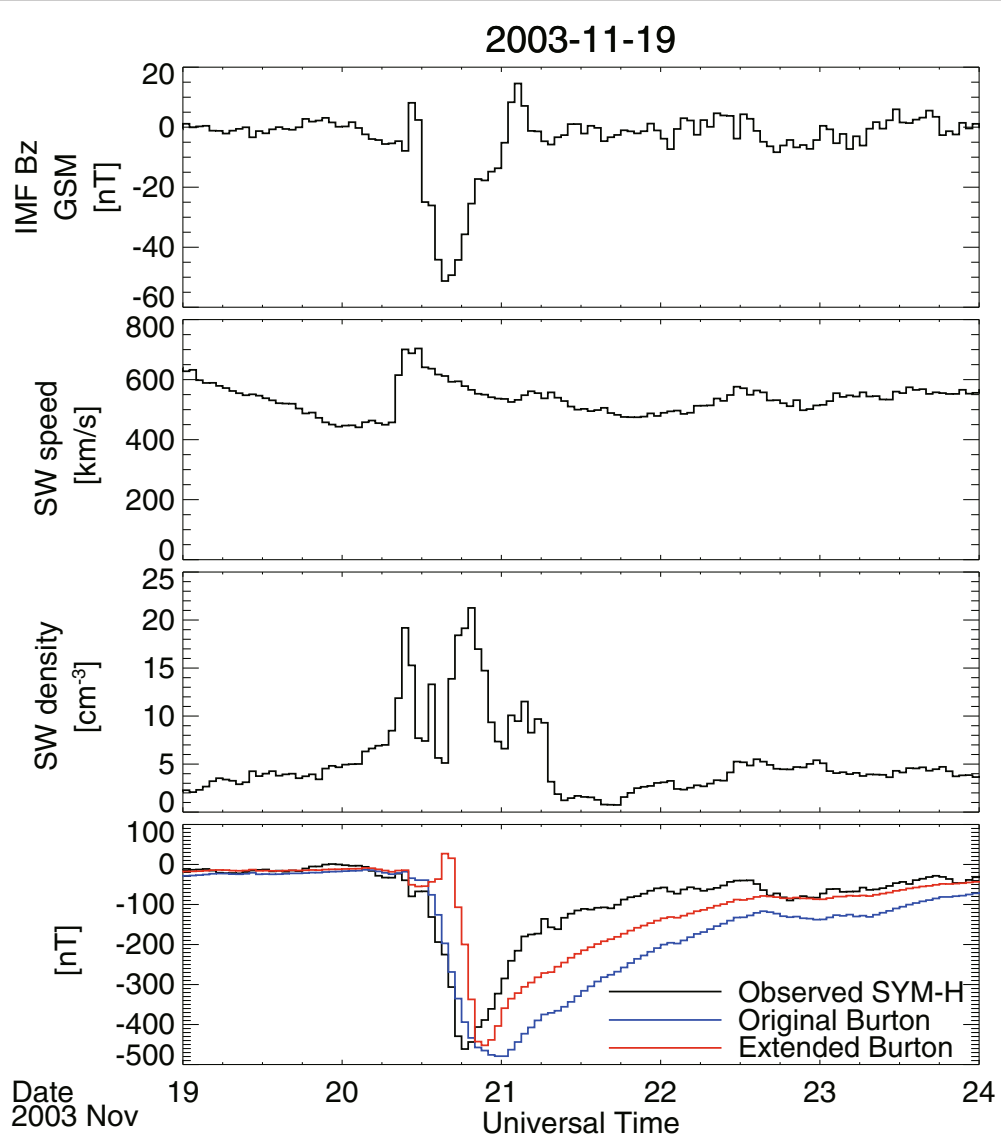

Figure 3 Same as Figure 2 except for the November 2003 storm. IMF, interplanetary magnetic field; Bz, the Z component of the magnetic field; GSM, geocentric solar magnetospheric; SYM-H, symmetric disturbance field in the horizontal direction. 


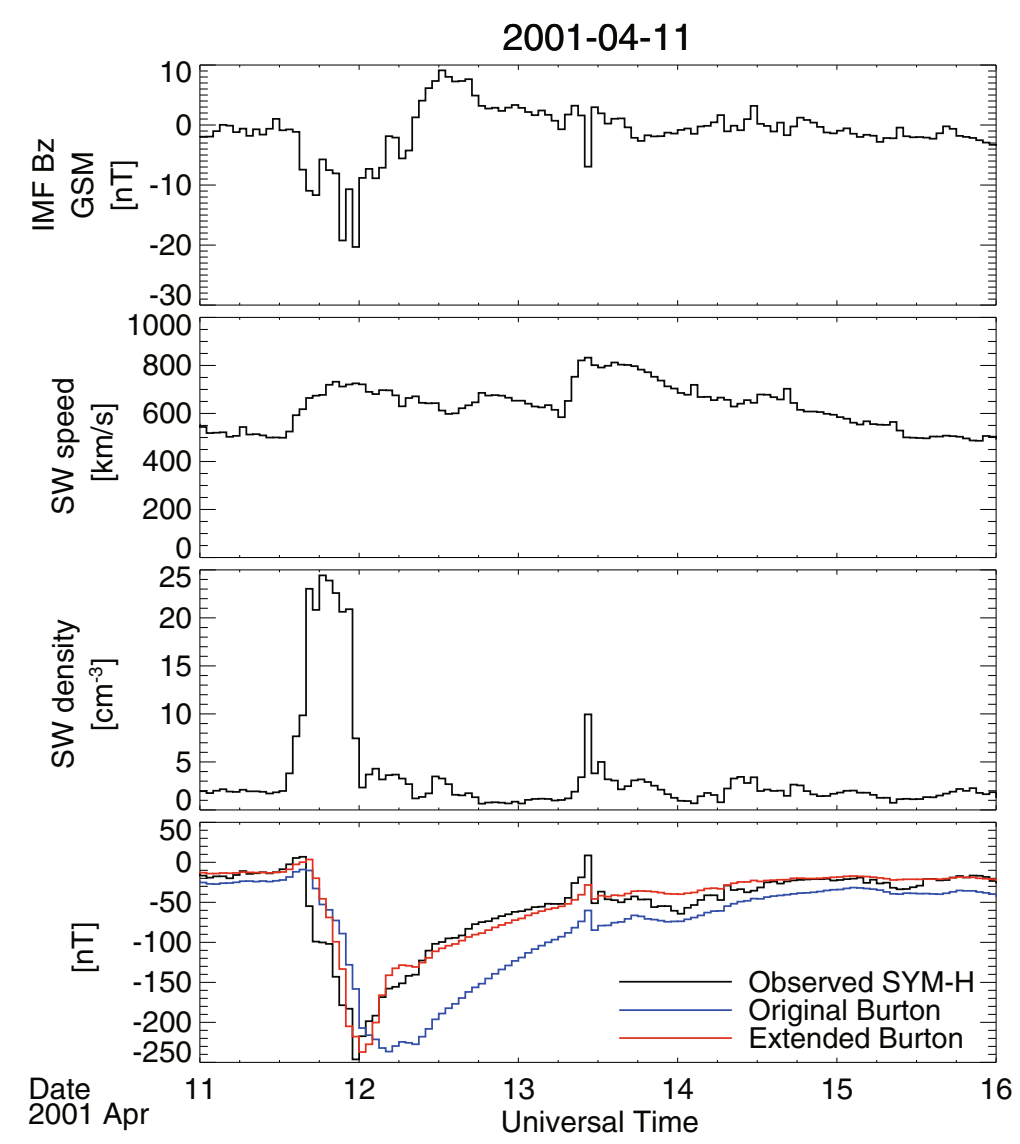

Figure 4 Same as Figure 2 except for the April 2001 storm. IMF, interplanetary magnetic field; Bz, the Z component of the magnetic field; GSM, geocentric solar magnetospheric; SYM-H, symmetric disturbance field in the horizontal direction.

For all events, the SYM-H profile derived from the original Burton's model does not show rapid recovery similar to the observed SYM-H. Our extended model, however, displays rapid recovery; the rapidness of the early recovery is comparable to that of the observed SYM-H. The extended model also reproduces two-step recovery. For the March 2001 event, the original Burton's model displays two-step development rather than two-step recovery; our extended model shows rapid development followed by rapid recovery.

All events except for the November 2004 event display an increase in solar wind density almost simultaneously with an increase in the solar wind speed and a decrease in IMF Bz. The enhanced density reduced down to the pre-event level in 12 to $24 \mathrm{~h}$, that is, when or before the IMF $\mathrm{Bz}$ negative excursion recovers to the pre-event level. These features indicate enhanced density in the magnetic cloud generated by a CME event. Such a density bump plays the key role in the extended model producing rapid recovery.

For the November 2004 event, a density bump appears in front of the southward IMF region (probably the sheath region of the interplanetary shock). IMF was northward during the period of the density enhancement. Since plasma entry across the flank-side magnetopause needs longer time than transport along the filed lines reconnected at the dayside magnetopause, the dense solar wind plasma could have spent a longer time to enter into the plasma sheet than the southward IMF cases.

\section{Modeling of the Carrington recovery}

We examine what SYM-H time profile the extended model produces with solar wind inputs that could cause the Carrington event. Figure 6 shows previously modeled solar wind inputs (speed, IMF Bz, and density) and the SYM-H time profiles estimated by the original Burton's model and our extended model. We used the best fit parameter pair obtained for the November 2004 storm. The solar wind speed and IMF $\mathrm{Bz}$ are the same as those used by Li et al. (2006) except for the duration of southward IMF. IMF $\mathrm{Bz}$ is $2 \mathrm{nT}$ before the event, followed by a decrease down to $-65 \mathrm{nT}$ and recovery to $2 \mathrm{nT}$. The duration of southward IMF is $2 \mathrm{~h}, 1 \mathrm{~h}$ longer than that used by $\mathrm{Li}$ et al. (2006). The solar wind speed is $450 \mathrm{~km} / \mathrm{s}$ before the event, increases to 


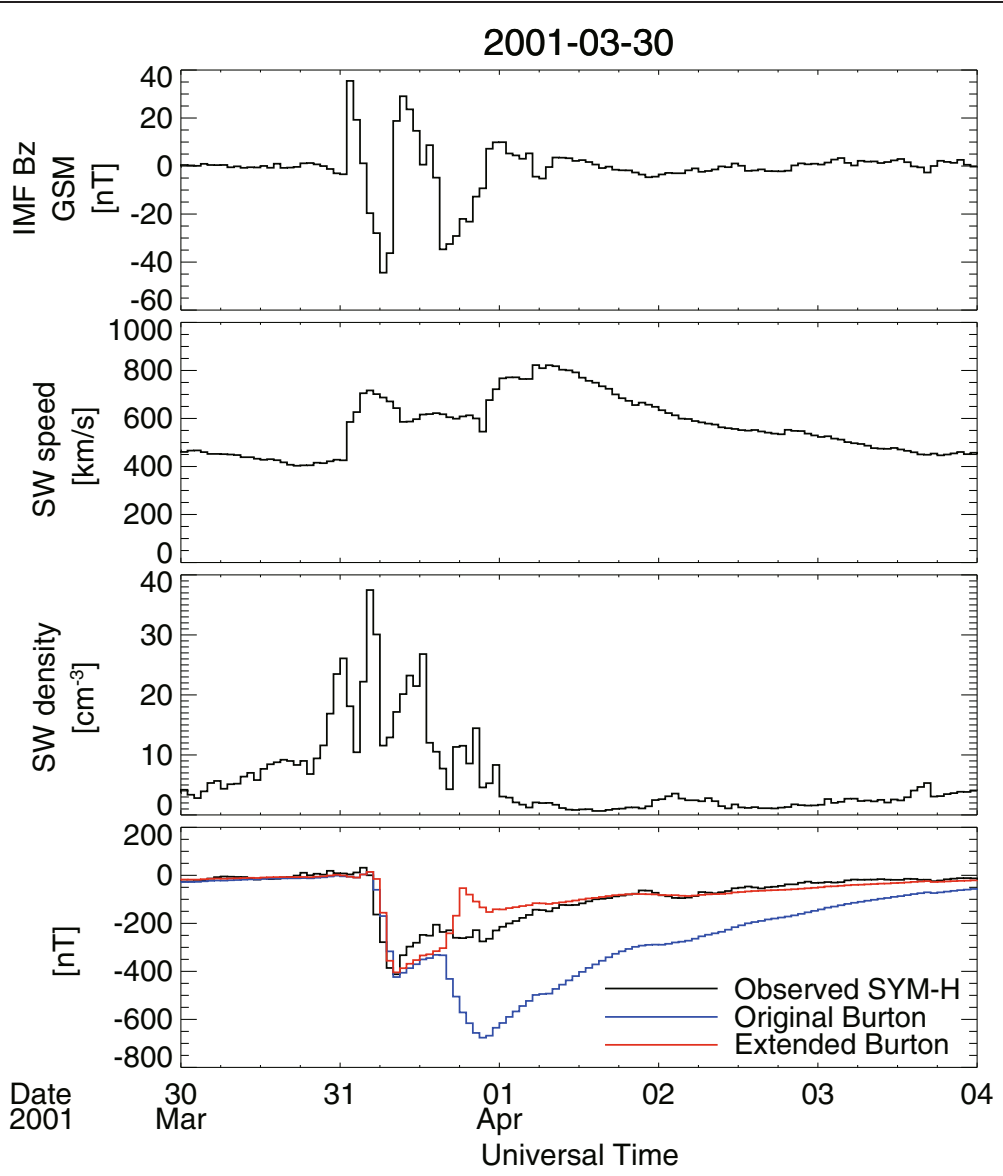

Figure 5 Same as Figure 2 except for the March 2001 storm. IMF, interplanetary magnetic field; Bz, the Z component of the magnetic field; GSM, geocentric solar magnetospheric; SYM-H, symmetric disturbance field in the horizontal direction.

$1,500 \mathrm{~km} / \mathrm{s}$, and recovers to $450 \mathrm{~km} / \mathrm{s}$. We used $\Delta t_{\mathrm{r}}=1 \mathrm{~h}$ and $\Delta t_{\mathrm{d}}=4 \mathrm{~h}$.

We chose the density profile such that the density enhancement appears in the early part of the southward IMF interval; this is a reasonable choice because all of the four intense storms examined in this study were accompanied by a density enhancement during the part/entire interval of strong southward IMF (see Figure 2). The density is $5 \mathrm{~cm}^{-3}$ except for a 1 -h bump of $100 \mathrm{~cm}^{-3}$.

Our model derived a SYM-H time profile showing a rapid recovery in the time scale comparable to the storm development. The original Burton's model did not reproduce such a rapid recovery. Figure 7 presents the SYM-H profiles derived from the original Burton's model and our model with the best fit parameter set obtained for each storm event examined in this study. The parameter pair is denoted at the top of each panel. Although the storm maximum level (i.e., SYM$H$ minimum) differs among cases, it is noted that our extended model reproduced two-step recovery for all cases.

\section{Preferred conditions for the flow-out effects}

The flow-out process is effective in decaying the ring current when (1) plasma sheet ions injecting into the inner magnetosphere are more tenuous than ring current ions and (2) drift trajectories encounter the dayside magnetopause. These conditions require (i) a decrease in plasma sheet density and (ii) strong and prolonged $\mathrm{E} \times \mathrm{B}$ convection. Since plasma sheet density is correlated with solar wind density (Terasawa et al. 1997; Borovsky et al. 1998; Ebihara and Ejiri 2000), a decrease in solar wind density is needed. For the continuous $\mathrm{E} \times \mathrm{B}$ drift, the dawn-dusk convection electric field needs to remain strong, and thus, solar wind speed needs to keep fast and/or the IMF southward component remains large. In summary, the flow-out effect is significant when solar wind density decreases during a period of fast solar wind and/or southward IMF.

For three of the four storm events examined in this study (the November 2003, April 2001, and March 2001 storm), an increase in solar wind density occurs during the earlier part of a period of strong southward IMF, and 
Table 1 Hourly averaged SYM-H (minimum), IMF Bz (minimum), solar wind density (maximum), $\left[a_{0}, \beta_{0}\right]$, and $\Delta t_{r}$

\begin{tabular}{|c|c|c|c|c|c|c|}
\hline & $\mathrm{SYM}-\mathrm{H}_{\min }[\mathrm{nT}]$ & $I \mathrm{MF} \mathrm{Bz}_{\mathrm{GSM} \min }[\mathrm{nT}]$ & $N_{\text {SWmax }}\left[\mathrm{cm}^{-3}\right]$ & $a_{0}$ & $\beta_{0}$ & $\Delta t_{\mathrm{r}}[\mathrm{hr}]$ \\
\hline November 2004 & -379.3 & -44.7 & 60.3 & -0.8 & -0.04 & 12 \\
\hline November 2003 & -461.3 & -51.3 & 26.5 & -1.0 & -0.25 & 0 \\
\hline April 2001 & -246.3 & -20.3 & 30.4 & -0.9 & -0.19 & 2 \\
\hline March 2001 & -412.0 & -44.4 & 37.5 & -1.4 & -0.23 & 1 \\
\hline
\end{tabular}

the enhanced density decreases during the later part. IMF was southward even after the density peak. For the November 2004 event, solar wind density sharply increases before IMF turns southward; we speculate that plasma sheet density becomes high before the convection electric field becomes large. We believe that solar wind structures with a density enhancement only in the earlier part of or prior to a southward IMF period are not unusual.

Our estimates of the Carrington recovery based on the extended Burton's model suggest that a solar wind density decrease by approximately $100 \mathrm{~cm}^{-3}$ is high enough to produce the observed rapid recovery. We believe that the number is more reasonable than a density of approximately 2,000 $\mathrm{cm}^{-3}$ estimated by $\mathrm{Li}$ et al. (2006), who proposed that such a huge increase in solar wind density drastically increases the magnetopause current and in turn recovers the Dst/SYM-H index quickly.

\section{Response time of plasma sheet density}

For three of the four storm events examined in this study (the November 2003, April 2001, and March 2001 storm), the best fit $\Delta t_{\mathrm{r}}$ was $\leq 2 \mathrm{~h}$. The quick response is supported by the density bump that occurred during a period of southward IMF. We believe that the dense solar wind that entered into the plasma sheet through

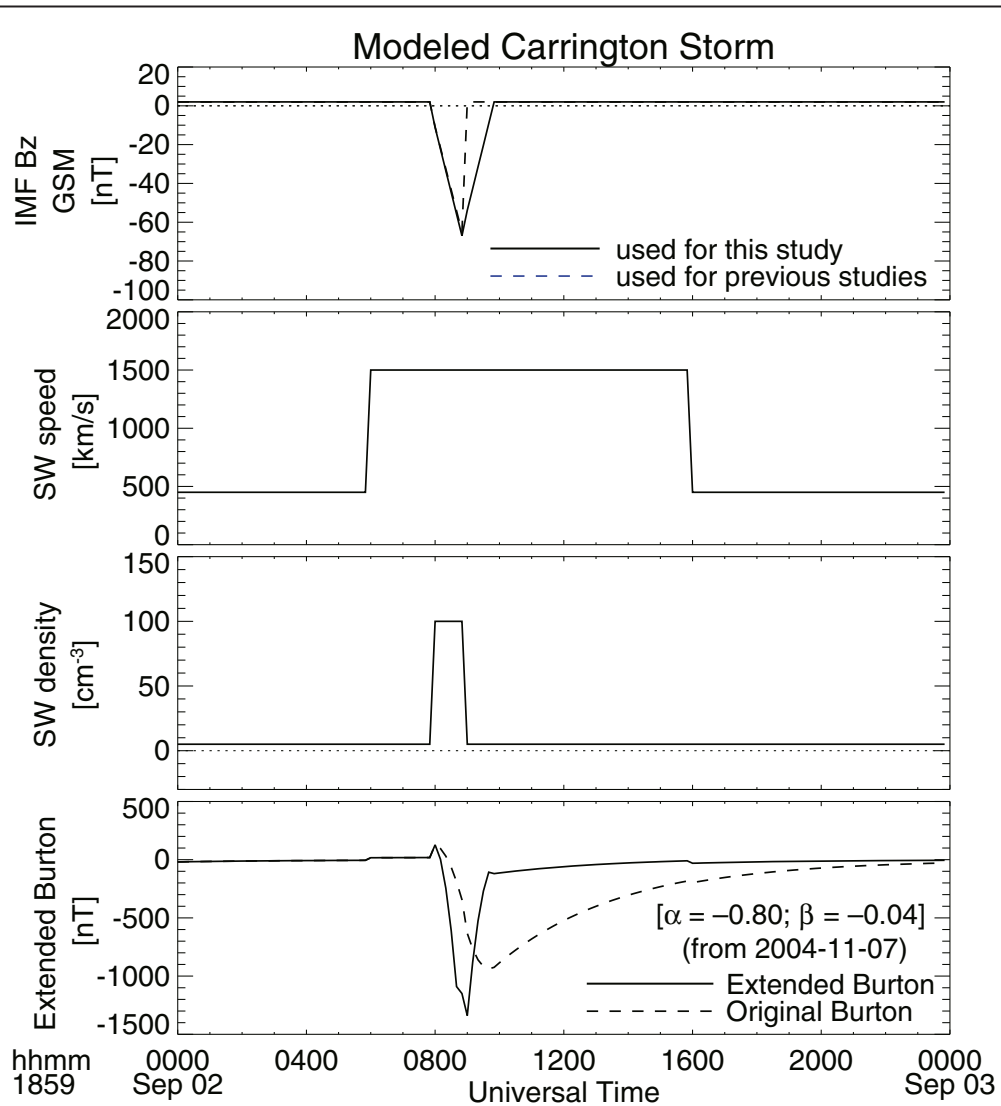

Figure 6 Solar wind conditions and the SYM-H time profiles estimated from the original Burton's model and our model. We used the parameter pair that best fits the observed SYM-H for the November 2004 storm. The solar wind inputs are almost the same as those estimated by Tsurutani (2003), Siscoe et al. (2006), and Li et al. (2006) except for the duration of southward IMF. A longer duration was used for this study. IMF, interplanetary magnetic field; Bz, the Z component of the magnetic field; GSM, geocentric solar magnetospheric; SYM-H, symmetric disturbance field in the horizontal direction. 


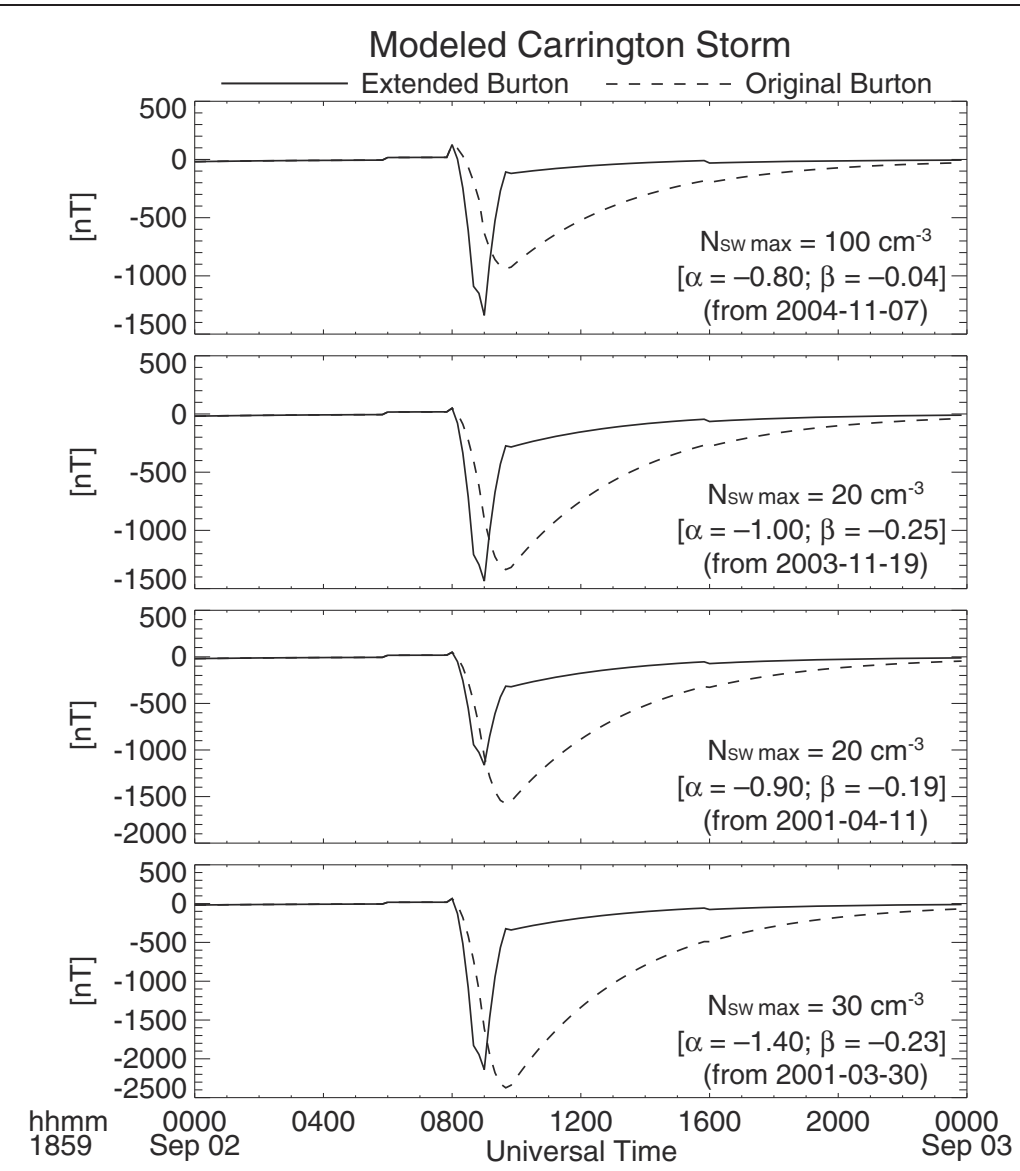

Figure 7 SYM-H time profiles of the Carrington storm modeled by the original Burton's model and our model. We used each pair of the best fit parameters derived for the intense storms examined.

the reconnected field lines convected at a speed comparable to the magnetosheath solar wind. For the November 2004 storm, on the other hand, we needed to adopt the response time of $12 \mathrm{~h}$ to best fit our model to the observed SYM-H. This is probably because the dense solar wind took a long time to enter into the plasma sheet under the condition of northward IMF. We assume plasma transport across the flank-side magnetopause due to instabilities such as the Kelvin-Helmholtz instability caused by flow shear at the magnetopause boundary. A larger number of intense storm events are necessary to identify the typical response time and/or its relationship with other solar wind parameters such as solar wind speed and IMF directions.

\section{Effects of ionospheric outflow}

Outflow of ionospheric ions into the magnetotail is enhanced after an interplanetary shock hit the magnetosphere and when energetic ions/electrons precipitate into the ionosphere particularly during substorms (e.g., Moore et al. 1999). Such outflowing ionospheric ions increase plasma density. This effect was not taken into consideration in our model. This study is the first step to incorporate the flow-out effect associated with solar wind density variations into Burton's model. It is well accepted that plasma sheet density is statistically well correlated with solar wind density (e.g., Terasawa et al. 1997). Effects of ionospheric outflow will be addressed in our future models.

\section{Ring current decay proportional to ring current energy content}

O'Brien and McPherron (2000) found a correlation between $\tau$ and $V B_{\mathrm{s}}$ and suggested that $V B_{\mathrm{s}}$ controls the position of the ring current. They assumed that the position is controlled by the convection electric field, which is correlated with the solar wind electric field. As the convection electric field increases, ring current ions are transported closer to the Earth, and the charge exchange loss rate increases because of higher density of the geocorona. We chose a constant value of $\tau$ for our model in this study to focus on evaluating whether the flow-out 
effect due to solar wind density decrease contributes to ring current rapid decay. We used a typical loss time scale of the most general, best accepted ring current ion loss process, charge exchange with the geocorona at $L=3$ to 4 . Time-varying $\tau$ will be incorporated in our future studies.

Wang et al. (2003) and Xie et al. (2008) proposed $\tau$ as a function of $P_{\mathrm{d}}$ for northward IMF and attributed the correlation to the size of the magnetosphere. The new loss term of our model depends on $\Delta N_{\mathrm{SW}}$ and in turn $\Delta P_{\mathrm{d}}$. We believe that the flow-out effect expressed by the new loss term can be distinguished from the effect of a change in the magnetospheric size. The change does not affect the densities of both injected and outflowing ions but the energy of outflowing ions, because the magnetic field strength adjacent to the dayside magnetopause changes.

\section{Conclusions}

We examined intense geomagnetic storms in order to address an open question: why did the Carrington storm recover very rapidly ( $>800 \mathrm{nT}$ in a few hours)? We tested a hypothesis that the rapid recovery is due to the flow-out of energetic ions that generate the westward ring current, by extending Burton's model. We added a new term representing variations of solar wind density which is correlated with plasma sheet density. We then applied the extended model to previously observed intense magnetic storms. Plasma sheet density variations make the flow-out process effective under the condition where the convection electric field remains strong. When the density drops, energetic ions contributing to the ring current flow out through the dayside magnetopause and are replaced by low-density plasma transported from a tenuous plasma sheet. We compared the time profile of the observed SYM-H (SYM-Hx $\left.{ }_{\text {obs }}\right)$ with that derived from our extended model $\left(\mathrm{SYM}-\mathrm{H}_{\mathrm{ext}}\right)$. We performed forward modeling to find the best fit of SYM$\mathrm{H}_{\text {ext }}$ to $\mathrm{SYM}-\mathrm{H}_{\text {obs. }}$. The best fit $\mathrm{SYM}-\mathrm{H}_{\text {ext }}$ displayed more rapid recovery than SYM-H based on the original Burton's model. Furthermore, our model reproduced a two-step recovery, a rapid initial recovery followed by a slow one. We also applied the best fit parameters to the Carrington event, utilizing solar wind data used by $\mathrm{Li}$ et al. (2006). Our model produced rapid recovery similar to the Carrington event recovery when the solar wind density increases up to approximately $100 \mathrm{~cm}^{-3}$ and suddenly drops under a condition of continuous southward IMF of $65 \mathrm{nT}$ and solar wind speed of 1,500 km/s. Such a solar wind density enhancement, as well as the IMF strength and the solar wind speed, is not beyond the observed limit. We conclude that the flow-out effect plays a significant role in the rapid recovery of the Carrington storm.

\section{Abbreviations}

Bz: the Z component of the magnetic field; CME: coronal mass ejection; GIC: ground-induced current; GSM: geocentric solar magnetospheric; IMF: interplanetary magnetic field; SYM-H: symmetric disturbance field in the horizontal direction.

\section{Competing interests}

The authors declare that they have no competing interests.

\section{Author' contributions}

KK proposed the topic, the new empirical model, and the analysis method and also performed the analysis. YE and RK collaborated with KK in discussing the analysis method and results and constructing the manuscript. All the authors read and approved the final version of the manuscript.

\section{Acknowledgements}

We thank Shin'ya Nakano of the Institute of Statistical Mathematics for helpful discussion. The SYM-H index was provided by the Data Analysis Center for Geomagnetism and Space Magnetism in Kyoto, Japan. OMNI solar wind data were provided through OMNI Web Plus (http://omniweb.gsfc. nasa.gov/). Part of the work of KK was done at the ERG-Science Center operated by ISAS/JAXA and STEL/Nagoya University. This work was supported by the Ministry of Education, Culture, Sports, Science and Technology (MEXT), Japan Society for the Promotion of Science (JSPS), Grant-in-Aid for Young Scientists (B) (grant \#26800257).

\section{Author details}

'Solar-Terrestrial Environment Laboratory, Nagoya University, Nagoya 464-8601, Japan. ${ }^{2}$ Research Institute for Sustainable Humanosphere, Kyoto University, Kyoto 611-0011, Japan. ${ }^{3}$ National Institute for Polar Research, Tachikawa 190-8518, Japan.

Received: 4 November 2014 Accepted: 17 April 2015 Published online: 08 May 2015

\section{References}

Aguado J, Cid C, Saiz E, Cerrato Y (2010) Hyperbolic decay of the Dst Index during the recovery phase of intense geomagnetic storms. J Geophys Res 115:A07220, doi:10.1029/2009JA014658

Alexeev II, Belenkaya ES, Kalegaev W, Feldstein YI, Grafe A (1996) Magnetic storms and magnetotail currents. J Geophys Res 101:7737-7747, doi:10.1029/95JA03509

Araki T (2014) Historically largest geomagnetic sudden commencement (SC) since 1868. Earth Planets Space 66:164, doi:10.1186/s40623-014-0164-0

Bolduc L (2002) GIC observations and studies in the Hydro-Québec power system. J Atmos Solar Terrestrial Phys 64:1793-1802, doi:10.1016/S1364-6826(02)00128-1

Borovsky JE, Thomsen MF, Elphic RC (1998) The driving of the plasma sheet by the solar wind. J Geophys Res 103(A8):17617-17639

Burton RK, McPherron RL, Russell CT (1975) An empirical relationship between interplanetary conditions and Dst. J Geophys Res 80:4204-4214, doi:10.1029/JA080i031p04204

Carrington RC (1859) Description of a singular appearance seen in the Sun on September 1, 1859. Mon Not R Astron Soc 20:13-15

Chen MW, Lyons LR, Schulz M (1994) Simulations of phase space distributions of storm time proton ring current. J Geophys Res 99:5745-5759, doi:10.1029/93JA02771

Cid C, Palacios J, Saiz E, Cerrato Y, Aguado J, Guerrero A (2013) Modeling the recovery phase of extreme geomagnetic storms. J Geophys Res Space Physics 118:4352-4359, doi:10.1002/jgra.50409

Cornwall JM, Coroniti FV, Thorne RM (1970) Turbulent loss of ring current protons. J Geophys Res 75:4699-4709, doi:10.1029/JA075i025p04699

Daglis IA, Thorne RM, Baumjohann W, Orsini S (1999) The terrestrial ring current: origin, formation, and decay. Rev Geophys 37:407-438, doi:10.1029/1999RG900009

Ebihara Y, Ejiri M (2000) Simulation study on fundamental properties of the storm-time ring current. J Geophys Res 105:15843-15859, doi:10.1029/1999JA900493

Ebihara Y, Ejiri M (2003) Numerical simulation of the ring current: review. Space Sci Rev 105:377-452, doi:10.1023/A:1023905607888 
Ebihara Y, Fok MC, Wolf MF, Thomsen MF, Moore TE (2005) Nonlinear impact of plasma sheet density on the storm-time ring current. J Geophys Res 110:A02208, doi:10.1029/2004JA010435

Ebihara Y, Fok MC, Immel TJ, Brandt PC (2011) Rapid decay of storm time ring current due to pitch angle scattering in curved field line. J Geophys Res 116:A03218, doi:10.1029/2010JA016000

Gonzalez WD, Tsurutani BT, Gonzalez ALC, Smith EJ, Tang F, Akasofu SI (1989) Solar wind-magnetosphere coupling during intense magnetic storms (1978-1979). J Geophysical Res 94:8835-8851, doi:10.1029/JA094iA07p08835

Gonzalez WD, Joselyn JA, Kamide Y (1994) What is a geomagnetic storm? J Geophys Res 99:5771-5792, doi:10.1029/93JA02867

Hamilton DC, Gloeckler G, Ipavich FM, Stüdemann W, Wilken B, Kremser G (1988) Ring current development during the great geomagnetic storm of February 1986. J Geophys Res 93:14343, doi:10.1029/JA093iA12p14343

Hodgson R (1859) On a curious appearance seen in the Sun. Mon Not R Astron Soc 20:15-16

Jordanova VK, Farrugia CJ, Thorne RM, Khazanov GV, Reeves GD, Thomsen MF (2001) Modeling ring current proton precipitation by electromagnetic ion cyclotron waves during the May 14-16, 1997, storm. J Geophys Res 106:7-22, doi:10.1029/2000JA002008

Keika K, Nosé M, Ohtani S, Takahashi K (2005) Outflow of energetic ions from the magnetosphere and its contribution to the decay of the storm time ring current. Space Physics 110(A9):1978-2012, doi:10.1029/2004JA010970

Kozyra JU, Fok MC, Sanchez ER, Evans DS, Hamilton DC, Nagy AF (1998a) The role of precipitation losses in producing the rapid early recovery phase of the Great Magnetic Storm of February 1986. J Geophys Res 103:6801-6814. doi:10.1029/97JA03330

Kozyra JU, Jordanova VK, Borovsky JE, Thomsen MF, Knipp DJ, Evans DS, McComas DJ, Cayton TE (1998b) Effects of a high-density plasma sheet on ring current development during the November 2-6, 1993, magnetic storm. J Geophys Res 103:26285. doi:10.1029/98JA01964

Langel RA, Estes RH (1985) Large-scale, near-field magnetic fields from external sources and the corresponding induced internal field. J Geophys Res 90:2487-2494, doi:10.1029/JB090iB03p02487

Li X, Temerin M, Tsurutani BT, Alex S (2006) Modeling of 1-2 September 1859 super magnetic storm. Adv Space Res 38:273-279, doi:10.1016/ j.asr.2005.06.070

Liemohn MW, Kozyra JU, Jordanova VK, Khazanov GV, Thomsen MF, Cayton TE (1999) Analysis of early phase ring current recovery mechanisms during geomagnetic storms. Geophys Res Lett 26:2845-2848, doi:10.1029/1999GL900611

Liemohn MW, Kozyra JU, Thomsen MF, Roeder JL, Lu G, Borovsky JE, Cayton TE (2001) Dominant role of the asymmetric ring current in producing the stormtime Dst*. J Geophys Res 106:10883-10904, doi:10.1029/2000JA000326

Monreal MacMahon R, Llop-Romero C (2008) Ring current decay time model during geomagnetic storms: a simple analytical approach. Ann Geophys 26:2543-2550, doi:10.5194/angeo-26-2543-2008

Moore TE, Peterson WK, Russell CT, Chandler MO, Collier MR, Collin HL, Craven PD, Fitzenreiter R, Giles BL, Pollock CJ (1999) lonospheric mass ejection in response to a CME. Geophys Res Lett 26:2339-2342

O'Brien TP, McPherron RL (2000) An empirical phase space analysis of ring current dynamics: solar wind control of injection and decay. J Geophys Res 105:7707-7719, doi:10.1029/1998JA000437

O'Brien TP, McPherron RL, Liemohn MW (2002) Continued convection and the initial recovery of Dst. Geophys Res Lett 29:2143, doi:10.1029/2002GL015556

Pulkkinen A, Bernabeu E, Eichner J, Beggan C, Thomson AWP (2012). Generation of 100-year geomagnetically induced current scenarios. Space Weather 10:S04003, doi:10.1029/2011SW000750

Siscoe GL, Formisano V, Lazarus AJ (1968) Relation between geomagnetic sudden impulses and solar wind pressure changes - an experimental investigation. J Geophys Res 73:4869-4874, doi:10.1029/JA073i015p04869

Siscoe G, Crooker NU, Clauer CR (2006) Dst of the Carrington storm of 1859. Adv Space Res 38:173-179, doi:10.1016/j.asr.2005.02.102

Terasawa T, Fujimoto M, Mukai T, Shinohara I, Saito Y, Yamamoto T, Machida S, Kokubun S, Lazarus AJ, Steinberg JT, Lepping RP (1997) Solar wind control of density and temperature in the near-Earth plasma sheet: WIND/GEOTAIL collaboration. Geophys Res Lett 24:935-938, doi:10.1029/96GL04018

Tsurutani BT, Gonzalez WD, Lakhina GS, Alex S (2003) The extreme magnetic storm of 1-2 September 1859. J Geophys Res 108:1268, doi:10.1029/ 2002JA009504
Turner NE, Baker DN, Pulkkinen TI, McPherron RL (2000) Evaluation of the tail current contribution to Dst. J Geophys Res 105:5431-5439, doi:10.1029/ 1999JA000248

Wang CB, Chao JK, Lin CH (2003) Influence of the solar wind dynamic pressure on the decay and injection of the ring current. J Geophys Res 108:1341, doi:10.1029/2003JA009851

Williams DJ (1981) Ring current composition and sources: an update. Planet Space Sci 29:1195-1203, doi:10.1016/0032-0633(81)90124-0

Xie H, Gopalswamy N, St Cyr OC, Yashiro S (2008) Effects of solar wind dynamic pressure and preconditioning on large geomagnetic storms. Geophys Res Lett 35:L06S08, doi:10.1029/2007GL032298

Yokoyama N, Kamide Y (1997) Statistical nature of geomagnetic storms. J Geophys Res 102:14215-14222, doi:10.1029/97JA00903

\section{Submit your manuscript to a SpringerOpen ${ }^{\odot}$ journal and benefit from:}

- Convenient online submission

- Rigorous peer review

- Immediate publication on acceptance

- Open access: articles freely available online

- High visibility within the field

- Retaining the copyright to your article

Submit your next manuscript at springeropen.com 